\title{
EMAS: Environment Monitoring and Smart Alert System for Internet of Things (IOT)
}

\section{Rabia Noor Enam}

Sir Syed University of Engineering \& Technology

Muhammad Tahir ( $\nabla$ tahirfattani@gmail.com )

Sir Syed University of Engineering and Technology https://orcid.org/0000-0003-3153-362X

\section{Syed Muhammad Nabeel Mustafa}

Sir Syed University of Engineering \& Technology

\section{Rukaiya Rukaiya}

Sir Syed University of Engineering \& Technology

\section{Research Article}

Keywords: Environmental Monitoring, Wireless Sensor Network, Monitoring applications, Active Node, Cluster based Routing

Posted Date: June 29th, 2021

DOl: https://doi.org/10.21203/rs.3.rs-562972/v1

License: (1) (1) This work is licensed under a Creative Commons Attribution 4.0 International License. Read Full License 
Noname manuscript No.

(will be inserted by the editor)

EMAS: Environment Monitoring and Smart Alert System for Internet of Things (IoT)

Rabia Noor Enam · Muhammad Tahir Syed Muhammad Nabeel Mustafa · Rukaiya Rukaiya

Received: date / Accepted: date

\section{Abstract}

The emergence of wireless sensor networks has enabled new classes of application for distributed systems that filter into many inter disciplinary fields. In the recent years, wireless technology has come up with great advancements and is supposed to be a great venture for the researchers. The recent advancements open up many significant energy optimization techniques in Internet of Things. The smart systems can now achieve paramount level of control of user comfort while reducing use of energy. Our main purpose is to construct smart Environment Monitoring and Surveillance system (EMAS). The system periodically measures temperature, light and humidity levels of the atmosphere. When a critical change in the environmental variables is detected, the EMAS system can notify the user via text message on their cell phone. Thus, they will be able to act to critical changes as quickly as possible and it may be able to intercept effects of the critical change. Results are obtained in the field tests reasonably ensuring that the proposed EMAS system possess excellent package delivery high power efficiency. The main goal of this paper is to bring more robustness and enhanced features in the wireless environment monitoring system.

Keywords Environmental Monitoring · Wireless Sensor Network · Monitoring applications · Active Node $\cdot$ Cluster based Routing

Muhammad Tahir

Sir Syed University of Eng. Tech. Karachi.

Tel.: +92-333-3688125

E-mail: tahirfattani@gmail.com

Extended author information available on the last page of the article

\section{Introduction}

The recent trends and modern technological development broaden the vision of Internet of Things (IoT). These advancements prevail viable smart solutions in terms of innovative applications. The distributed wireless communication networks increase the scope of surveillance by deploying cheap, low power consuming and multi-dimensional sensors that are tiny in size capable of transferring short range information [1][2]. The region of the environmental monitoring scheme exists in the bigger classes of distributed control implementation. Technological advances in microelectronic mechanical 
systems (MEMS) and wireless communication technologies have accelerated the growth of minute, low-cost, low-power and multi-purpose intelligent sensor nodes in a wireless sensor network (WSN). Installed in an open region, these smart sensor nodes are linked via internet wireless connections [3], as shown in figure 1 .

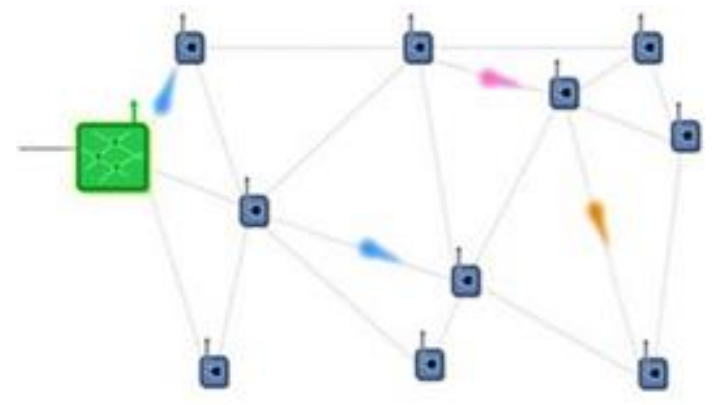

Fig 1. Wireless Sensor Network

The data information is transferred through the networks of nodes of the system in which node perform complex task such as statistical system. The advantage of implementing computing and networking capabilities is that, it allows sensor networks to be re programmed and re-tasked. An essential aspect of WSN application is environmental monitoring. It has grown extensively along with the latest technological development. Environmental monitoring system generally monitors and examine environmental parameters such as temperature, humidity, light [2][4][5]. The technology used for sensing controlling is combination of electric magnetic fields sensors, radio wave frequency sensors, optical electro-optics sensors, infrared sensors. Sensors work on multi hopped, short distance, energy efficient wireless links. Multi-hop routing introduced an important way for topology management and medium access control [6]. WSNs are capable of operating unattended in harsh climate where humanin-the-loop surveillance systems are unsafe ineffective [7]. The environment monitoring and smart alert system (EMAS) system is designed on a sensor network through which analyzing, monitoring and vigilance of environment sampling, aggregation of information and health of monitoring and vigilance of environment conditions can possibly be done by sensor nodes. The data obtained through this is transferred to base station (BS) in a multi hoping process thereby uploading the data to the server to obtain desired objectives as shown in figure 2 .

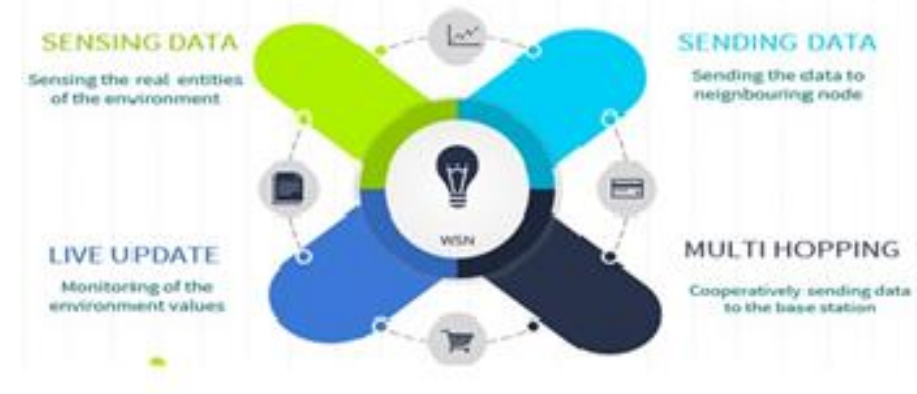

Fig. 2 Module Representation 
The rest of the paper is organized as follows: section 2 contains literature survey, section 3 explains the methodology, section 4 discusses results, findings and issues and section 5 concludes the paper.

\section{Literature Survey}

The advancements in wireless communication technologies and the development in IT are all set to open the gateways for the generation of cheap sensors. A lot of work is done on WSN in past making it more advance and applicable on a larger scale. Some of the work is described in this section. Wireless sensor networks are the fundamental building block of the environmental monitoring system [2]. Nodes show up their full strength when deployed in a network [8]. In severe circumstances, automation of the surveillance method can be useful. With WSN short-range transmission, agro climate in the Amazon was tracked. More remote nodes were discovered to bear a loss of performance, while nodes close the sink retained their level of performance [9]. By combining sensor technology IoT [10], the networks of environmental sensors will become a study instrument of future earth and environmental science systems [11]. The use of wireless sensing instruments was tested to monitor residential circumstances. It was discovered that setting up a sensor networks was simple, but complicated programming was needed to make it work [12]. A web-based environmental surveillance system was created to program wireless devices to send signals. The signals are then transmitted to the database in a code written in java. A web-based system is intended to enable the user to access information such as data type and sensor place [13]. We have designed a web and application based environmental monitoring and alert system which works on the principle of IoT with wireless sensors deployed to gather the certain data for the user. A user can monitor the conditions of the area, where sensors are deployed, on the mobile application and on the web.

The environmental monitoring and alert system consist of the capacities of both hardware and software. System hardware comprises of one or more processing and control units linked to the control unit, a set of peripheral devices. The control unit operates on the peripheral data provided or on the system's pre-defined guidelines. Commands are sent by ethernet cable, telephone lines, and fibreoptic cables to the peripherals from the central unit. Software plays an important role in running the hardware and logically linking all the units. It enables the control unit to control the peripheral device activities. The architecture used in EMAS is 'tiered architecture', with gateways, controllers and repeaters. A peer-to-peer (P2P) architecture surfaced. In P2P architecture, the devices failure doesn't affect the whole architecture but only one device. The sensor network architect for HVAC application can be tiered or flat. Lowest sensor nodes are the originators of the data of the sensor. HVAC energy usage depends on external environmental conditions [14]. The nodes are basically used for sensing purposes but they can perform general purpose computing networking. Nodes on the field are deployed in clusters, Traditional routing protocols for WSN are usually not optimal when it comes to energy consumption. Clustering is effective in saving the energy during the use of WSN [3]. Data is sent by sensor node to the cluster head, through the cluster in a multi-hop style. Multi hop routing is 
an important service required for WSN [15]. A cluster is a subset of vertices linked to the graph [16]. The head of the cluster transmits the sensor information.

\section{Methodology}

Our proposed model's design of EMAS System covers the entire building or subject area. It customizes the heating/cooling or other sensed parameter to the needs while conserving energy, just like an HVAC control system. HVAC system is specified for a building while EMAS System gives a generic platform to work in several other environmental monitoring systems as well. The setup and installation of an EMAS System test bed are described in this section. The sensor nodes, including their hardware and software designs and the actuator, which acts on the received data from the control unit, will be discussed.

\section{a. Sensor Network Node}

We used UC Berkley/Crossbow motes as sensor nodes in our deployment. Mica2 (shown in Table 1), these members of the mote family, use several bidirectional channels, for example $433 \mathrm{MHz}$ with the data rate of 38/19Kbps. Atmel Atmega128 microprocessor running at $7.3728 \mathrm{Mhz}$, with around $512 \mathrm{~KB}$ of storage. The required voltage is provided by a pair of standard AA batteries, while other renewable energy sources can also be employed.

\begin{tabular}{|c|c|c|c|c|c|c|}
\hline \multicolumn{2}{|c|}{ Mote type } & WeC & \begin{tabular}{l|l} 
Rene & Rene2 \\
\end{tabular} & Mica & Mica2 & Mica2Dot \\
\hline \multicolumn{7}{|c|}{ Example picture } \\
\hline \multirow{4}{*}{ MCU } & Chip & AT90L58535 & ATmega163L & ATmega103t & \multicolumn{2}{|c|}{ ATmega128L } \\
\hline & Type & $4 \mathrm{MHz}, 8$ bit & $4 \mathrm{MHz}, 8 \mathrm{bit}$ & $4 \mathrm{MHz}, 8$ bit & \multicolumn{2}{|c|}{$8 \mathrm{MHz}, 8 \mathrm{bit}$} \\
\hline & $\begin{array}{l}\text { Program memory } \\
\text { (KB) }\end{array}$ & 8 & 16 & 128 & \multicolumn{2}{|c|}{128} \\
\hline & RAM (KB) & 0.5 & 1 & 4 & \multicolumn{2}{|c|}{4} \\
\hline \multirow{3}{*}{$\begin{array}{c}\text { External } \\
\text { nonvolatile } \\
\text { storage }\end{array}$} & Chip & \multicolumn{2}{|c|}{$24 L C 256$} & \multicolumn{3}{|c|}{ AT4SDB014B } \\
\hline & Connection type & \multicolumn{2}{|c|}{$12 \mathrm{C}$} & \multicolumn{3}{|c|}{ SPI } \\
\hline & Size (KB) & \multicolumn{2}{|c|}{32} & \multicolumn{3}{|c|}{512} \\
\hline \multirow{2}{*}{$\begin{array}{l}\text { Defaut } \\
\text { power } \\
\text { source }\end{array}$} & Type & Coin cell & \multicolumn{3}{|c|}{$2 \times A 4$} & Coin cell \\
\hline & $\begin{array}{c}\text { Typical capacity } \\
\text { (mAh) }\end{array}$ & 575 & \multicolumn{3}{|c|}{2850} & 1000 \\
\hline \multirow{4}{*}{ RF } & Chip & \multicolumn{3}{|c|}{ TR1000 } & \multicolumn{2}{|c|}{ CC1000 } \\
\hline & Radio frequency & \multicolumn{3}{|c|}{$868 / 916 \mathrm{MHz}$} & \multicolumn{2}{|c|}{$\begin{array}{c}868 / 916 \mathrm{MHz}, 433, \\
\text { of } 315 \mathrm{MHz}\end{array}$} \\
\hline & Raw speed (kbps) & \multicolumn{2}{|c|}{10} & 40 & \multicolumn{2}{|c|}{38.4} \\
\hline & Modulation type & \multicolumn{2}{|c|}{ On/Oft key } & $\begin{array}{l}\text { Amplitude } \\
\text { Shift key }\end{array}$ & \multicolumn{2}{|c|}{$\begin{array}{l}\text { Frequency } \\
\text { Shitt key }\end{array}$} \\
\hline
\end{tabular}

Table 1. Family of Motes

Due to their small sizes and wireless communication capabilities, we were able to deploy motes in remote regions with minimum interference. Table 1 shows the specifications of the motes we used in our model.

TinyOS, and nesC, are component based OS and programming language (respectively) for systems with embedded networks, provide a strong software foundation on which to create applications. We chose the UC Berkeley platform running TinyOS because of hardware and software availability, as 
well as a similar match with the system requirements and Mica2 motes' features. Sleep states of low power are achieved by the TinyOS low-level hardware functionality.

In most circumstances, the rates of application data are quite low, which let the sensor nodes to sleep for the most of the time and just sample, compute, and transmit on a periodic basis. In sleep mode, you can save energy by disconnecting the sensors, turning off the radio and making the processor to go to sleep. Because current usage is assessed in milliamps (mA) during sleep, battery life is greatly extended. As a result, the most of the time, the draw of current is very low, with short-duration spikes during transmitting, processing and receiving data. As a result, the life of battery was increased; but, due to the current surges, the battery capacity was reduced.

On the Mica2, the Chippon (CC1000) radio is a single-chip UHF transceiver developed such wireless applications that are low in power and voltage. It can operate on many channels, making it ideal for network protocols that want to use frequency hopping. However, to avoid adjacent channel interference, the neighboring channel spacing should be more than $150 \mathrm{KHz}$. The radio is highly programmable and, among other things, allows for the changing of output power levels. On ADC channel 10, the measurement of the received signal intensity is also provided, known as RSSI.

This feature is available to any TinyOS program that wishes to sample the signal strength. Another crucial deployment element is that, the radio has a very sensitive receiver that can be interfered with by another Mica2 node's near local oscillator. To avoid local oscillator interference, a minimum distance of 2 feet must be maintained between nodes. The application-specific communication protocols' implementation of is less complex than what a full-fledged system would require. In a single-hop broadcast network, each sensor functions as a transmit-only device. From the sensor board's temperature sensor, the node periodically collects temperature data, makes a bundle of entries and then, schedules a transmission. The control unit receives the information, analyses it and sends it to EMS. The control unit may choose to perform an actuation and send a message to the actuation node as part of its processing. In the following part, we go over the specifics of the actuation node's implementation.

\section{b. Sensor Board}

We chose the "MIB520 USB Interface Board" as our sensor peripheral to the sensor node based on the requirements of our application. The following sensors are included in the sensor board:

1. A light photo resistor (Clairex CL9P4L) [13]

2. A thermistor (YSI 44006) [23]

3. A humidity or temperature sensor

4. A Barometric pressure or temperature sensor

5. Light sensor

6. 2-Axis accelerometer

7. Global Positioning System 
The 10 bit ADCs on the sensor board are used to sample the data from these sensors. Because these sensors are highly nonlinear, calibration is difficult. The board also includes a large prototyping space for the usage of additional sensors and actuators. The sensor board, in order to save power, operates at low duty-cycles and has low sampling speeds. The ADC measures the voltages that have been split. It takes roughly $10 \mathrm{~ms}$ to start up and draws $1.235 \mathrm{~mA}$ of current. The thermistor has a precision of roughly 0.04 degrees Celsius and a starting time of roughly 10 milliseconds. The noise in the system reduces measurement precision to roughly 0.5 degree Celsius. The accuracy degrades at the extremes because the thermistor curve is not linear. The sensor boards are changeable, ensuring that physical phenomenon readings are precise to within the sensor precision limits. Because the input from the sensors is analyzed, a signal created based on this data regulates cooling/heating and airflows in the air-ducts, changeability and precision are critical for HVAC applications.

Since the sensor board is used in such a low-duty-cycle application, it's likely that the startup time of each sensor dominates power consumption. The startup time is the amount of time it takes for a sensor to stabilize its reading once it has been powered up. Many sensor makers expect that the sensor will be powered up once and then remain on permanently. In low-power applications where the platform is powered by batteries that must last a long time, sensors must turn on and off quickly without consuming too much current. The sensors have a very low duty cycle and sample rate because temperature variations in an air mass, such as an office space, occur over long periods of time. For our application, the sensors were powered for a few milliseconds every 1.5 minutes. Sensors with a long initialization time (up to a few seconds) need more current for a longer period of time, resulting in higher power consumption.

\section{c. Actuation Board}

MDA 100 is the actuation node that we used. The actuation node's job is to receive messages from the control unit and respond by altering the HVAC system's functioning. The temperature in the simulated office area was relayed via the messages, which had to be sent on to one of the EMAS system's control units in charge of heating and cooling.

The MDA 100 series sensor boards incorporate a precise thermistor, a light sensor/photocell, and a general prototyping area. The prototype area supports all eight channels of the Mote's analogue to digital converter (ADCO-7), both USART serial ports, and the 12C digital communications bus .

The Mica2's Atmega128 has access to four hardware timers, numbered 0-3. TinyOS uses Timer0, one of the four timers, to provide a software abstraction of Timers to any app that wants to use it. Timer 1 is used exclusively by the Chipcon radio as part of the Radio Timing module to sync a packet receiver to the transmitter, while Timer2 is used by the SpiByteFifo module to provide a byte-level abstraction to the radio, shifting bits out when transmitting and shifting bits in when receiving. The only remaining timer is Time3. The problem faced was that we were receiving raw data from the control unit. The 
readings of temperature data were required to be translated into PWM (Pulse Width Modulated). This was required for any HVAC control system to perform according to the requirement of the system. Timer3 of Atmega128 microcontroller's, which is connected to pin 26 on the 51-pin connection was used. It has a resolution of up to 16 bits and hardware interrupts. In the Fast PWM mode, we used the onchip clock of 7.3728MHz to generate a PWM signal.

The PWM waveform must be converted to an analogue signal since the EMAS system wants an analogue value for the temperature input. To generate the signal, we created a basic low pass filter on the board. The base frequency of a typical PWM signal is set, but the pulse width is changeable. The frequency of the generated signal can be adjusted using the following formula

formula:

$$
F_{P W M}=f_{\text {req-clk }} /\left[A *\left(1+\text { Max }_{\text {timer }}\right)\right]
$$

- Where $\mathrm{f}_{\text {req-clk }}$ is the input frequency to the PWM module,

- Max $\mathrm{x}_{\text {timer }}$ is for the timer counter upto which it counts the maximum value of counts

- A can be chosen from any values among (2 to the power of $0,3,6,8$ or 10 ). It is the pre-scalar divider.

The PWM was run with the resolution set to 8 bits, therefore Max timer $_{\text {was }} 255$ and the A was set to $2^{\wedge} 3=8$. As a result, the PWM output frequency was set to $3.6 \mathrm{KHz}$.

The amplitude of the original unmodulated signal is precisely proportional to the pulse width. In other words, the frequency waveform of a PWM signal is constant, however the duty-cycle varies depending on the amplitude of the original signal.

\section{d. Control Module}

The EMAS system's control unit after reading the sensor node's generated data process to be recorded as the process variable $\mathrm{Vy}$. The $\mathrm{Vy}$ is fed back to system in the form of closed feedback loop of the actuation node.

The process and the controller are the two main components of the EMAS system. The manipulated variable, also known as the control variable, is the only input to the procedure. It is represented by $\mathrm{Vu}$. The set point value of the Vy is the desired value represented as Vsp. The difference between the two variables Vsp - Vy is control error Ce . The controller has one input i.e. Ce, and one output, i.e. Vu.

\section{e. MTS400/MTS420 Sensor Board}

Figure 3 shows the MTS400, which has five basic environmental sensors as well as a GPS module (MTS420). The features of these boards allow for a wide range of applications, from a simple wireless weather station to a large network of environmental monitoring nodes. Agriculture, industrial, forestry, HVAC, and other industries can all benefit from this technology. The most energy-efficient digital IC-based board-mount sensors are used in these environmental sensor boards. 
The MTS420's GPS module can be used to track freight, cars, vessels, and wildlife, as well as positioning identification of Motes put in inaccessible environments.

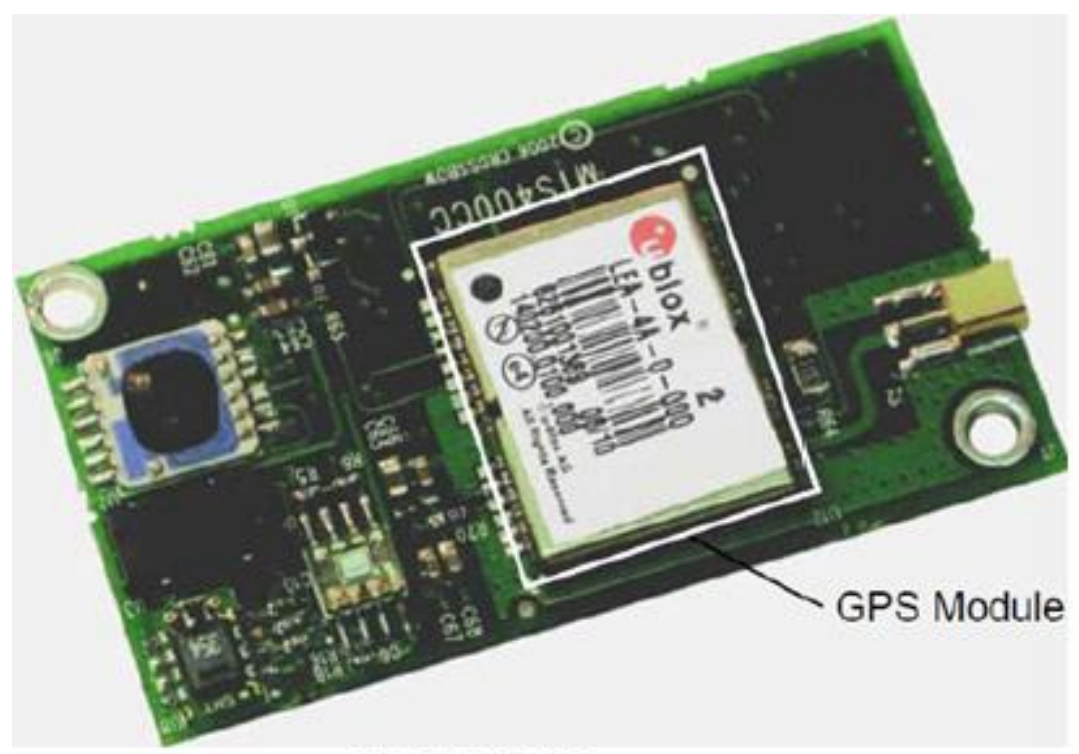

Fig 3. MTS420CC

\section{f. Humidity and Temperature Sensor}

The Sensirion SHT11 is a multi-sensor humidity and temperature module with a calibrated digital output on a single chip, as illustrated in Table 2. A 14-bit analog-to-digital converter and a serial port are built into the chip. SHT11s are calibrated individually.

\begin{tabular}{|c|c|c|}
\hline Sensor Type & \multicolumn{2}{|c|}{ Sensirion SHT11 } \\
\hline Channels & Humidity & Temperature \\
\hline Range & 0 to $100 \%$ & $-40^{\circ} \mathrm{C}$ to $80^{\circ} \mathrm{C}$ \\
\hline Accuracy & $\pm 3.5 \%$ RH (typical) & $\pm 2{ }^{\circ} \mathrm{C}$ \\
\hline Operating Range & \multicolumn{3}{|c|}{3.6 to 2.4 volts } \\
\hline Interface & \multicolumn{2}{|c|}{ Digital interface } \\
\hline
\end{tabular}

Table 2. Summary of Sensirion(B) SHT11's Specifications

A programmable switch controls the power of this sensor. A programmable switch is also used to enable the control interface signals. The humidity and temperature are converted to digital units using an analog-to-digital converter in the sensor.

\section{g. Barometric Pressure and Temperature Sensor}

The Intersema MS55ER is a hybrid SMD device with a piezo-resistive pressure sensor and an ADC interface Id. It converts pressure and temperature measurements into a 16-bit data word. All communications take place over a three-wire interface. The details are shown in Table 3 below: 


\begin{tabular}{|c|c|}
\hline Sensor Type & \begin{tabular}{c} 
Intersema MS5534 \\
\hline Channels
\end{tabular} \\
\hline Pressure and Temperature \\
\hline Accuracy & $\begin{array}{c}\text { Pressure: } 300 \text { to } 110 \mathrm{mbar} \\
\text { Temperature: }-10^{\circ} \mathrm{C} \text { to } 60^{\circ} \mathrm{C}\end{array}$ \\
\hline $\begin{array}{c}\text { Pressure: } \pm 3.5 \% \\
\text { Temperature: } \pm 2^{\circ} \mathrm{C}\end{array}$ \\
\hline Operating Range & 3.6 to 2.2 volts \\
\hline Interface & Digital interface \\
\hline
\end{tabular}

Table 3. Summary of the Intersema () MS55ER's Specifications

A programmable switch controls the power of this sensor. A programmable switch is also used to enable the control interface signals. The pressure and temperature are converted to digital values using an analog-to-digital converter in the sensor.

\section{h. Light Sensor}

The TLS2550 is a two-wire, 5M Bus serial interface digital light sensor. TAOS, Inc is the company that makes it. It provides light measurements over an effective 12-bit dynamic range by combining two photodiodes and a compounding analog-to-digital converter on a single CMOS integrated circuit.

\begin{tabular}{|c|c|}
\hline Sensor Type & Taos TSL2550 \\
\hline Channels & Light \\
\hline Range & $400-1000 \mathrm{~nm}$ \\
\hline Operating Range & 3.6 to 2.7 volts \\
\hline Interface & Digital interface \\
\hline
\end{tabular}

Table 4. Summary of TAOS TSL2550's Specifications

A programmable switch controls the power of this sensor. A programmable switch is also used to enable the control interface signals. The conversion from light to digital units is done via an analogto-digital converter in the sensor. Specifications of TAOS are defined in Table 4 above.

\section{i. Axis Accelerometer}

The accelerometer is a 2-axis MEMS surface micro-machined device with a weight of less than $2 \mathrm{~g}$. It has a very low current draw of only $1 \mathrm{~mA}$. Tilt detection, movement, vibration, and/or seismic measurement are all possible applications for the sensor. The sensor outputs are connected to the $\mathrm{ADCl}$ and $\mathrm{ADC} 2$ channels on the Mote. Table 5 explains the details of axis accelerometer.

\begin{tabular}{|c|c|}
\hline Sensor Type & Analog Devices ADXL202JE \\
\hline Channels & $X(A D C 1), Y(A D C 2)$ \\
\hline Range & $\pm 2 \mathrm{G}\left(1 \mathrm{G}=9.81 \mathrm{~m} / \mathrm{s}^{2}\right)$ \\
\hline Sensitivity & $167 \mathrm{mV} / \mathrm{G}, \pm 17 \%$ \\
\hline Resolution & $2 \mathrm{mG}(0.002 \mathrm{G}) \mathrm{RMS}$ \\
\hline Offset & VBATTERY/2 $\pm 0.4 \mathrm{~V}$ \\
\hline Operating Range & 3.6 to $3.0 \mathrm{~V}$ \\
\hline Interface & Analog interface \\
\hline
\end{tabular}

Table 5. Summary of the ADXL202JE's Specifications 


\section{j. $\quad$ GPS}

In the case of MTS420CA/CB, the GPS module utilized is Leadtek GPS-9546, while in the case of MTS420CC, it is uBloxLEA-4A. The GPS module's output is connected to the Mote's serial USART1 port. The module comes with an external, active antenna. The antenna is powered by the GPS module. Summary of various GPS Receivers is shown in Table 6. below:

\begin{tabular}{|c|c|c|}
\hline & MTS420CA/CB & MTS420CC \\
\hline GPS Module & Leadtek GPS-9546 & uBlox LEA-4A \\
\hline GPS Chipset & SiRFstarlle LP & ANTARIS 4 \\
\hline Channels & 12 & 16 \\
\hline Meters & $10 \mathrm{~m}, 2 \mathrm{D}$ & $3 \mathrm{~m}$ CEP \\
\hline Start Time (sec) & 45 Cold; 38 Warm; 8 Hot & 34 Cold; 33 Warm; 3.5 Hot \\
\hline Reacquisition Time & 0.1 sec (typical, w/o dense foliage) & $<1 \mathrm{sec}$ \\
\hline Protocol & NMEA-0183 and SIRF binary protocol & NMEA-0183 \\
\hline Current & 60 mA at 3.3 V & 35 mA at 3.3V \\
\hline Interface & \multicolumn{2}{|c|}{ Serial UART interface } \\
\hline Antenna & External active antenna, power supplied by GPS module \\
\hline
\end{tabular}

Table 6.Summary of the GPS Receiver Specifications

\section{k. Turning Sensors On and Off}

An analogue power switch at location U7 controls power to all of the sensors on the MTS400/420 sensor board. It can be programmed to turn on and off the power to specific sensors. The sensors are switched off by default. The sensor board's power demand is reduced as a result of this design.

\section{Iris Mote (XM2110)}

Crossbow Technology's IRIS is the latest generation of Motes. The Atmel RF230, an IEEE 802.15.4 compliant, ZigBee ready radio frequency transceiver combined with an Atmega1281 microcontroller, is used in the XM2110 (2400 to $2483.5 \mathrm{MHz}$ band), fig 4.

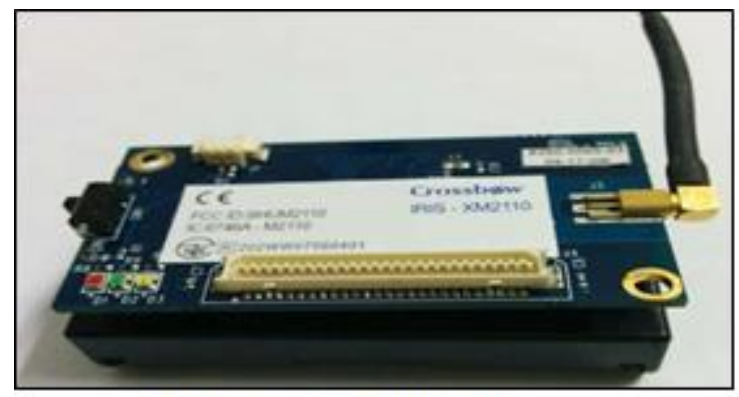

Fig 4. Iris Mote - XM2110

In comparison to prior generation MICA Motes, these upgrades give up to three times the radio range and double the program memory. All application software and sensor boards are compatible with the XM2110, which uses the same MICA family, 51 pin I/O connector, and serial flash memory. The block diagram of XM2110 is shown in fig 5. 


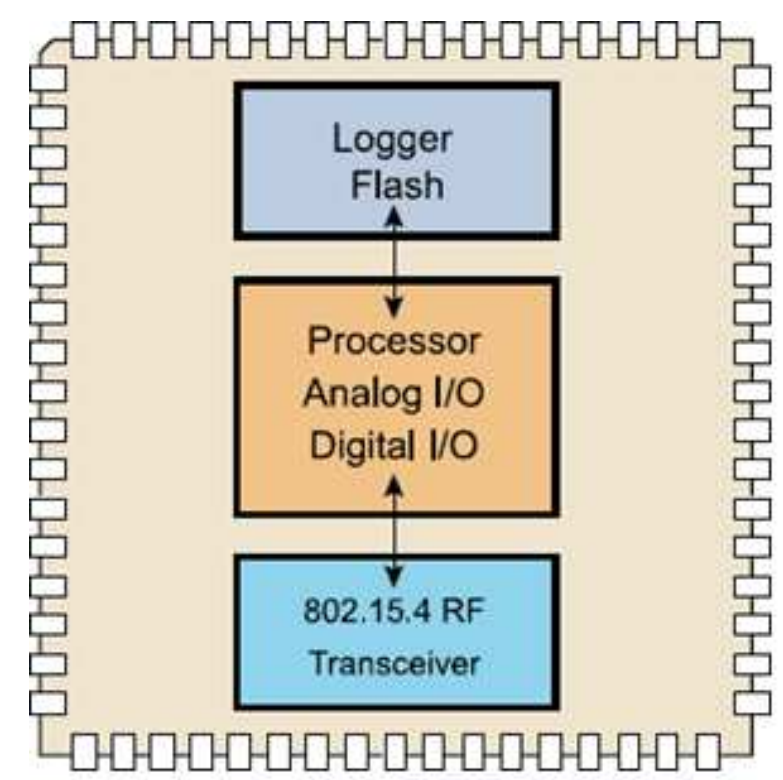

Fig 5. XM2110CB Block Diagram

\section{m. MIB520 USB Interface Board}

The MIB520 (fig 6) connects the IRIS and MICA families of Motes to the USB bus for communication and in-system programming. The USB bus is used to provide electricity to the devices. The male connector on the MIB520CB is male, while the other connector on the MIB520CA is female.

To program the Motes, the MIB520 features an on-board in-system processor (ISP) - an Atmega16L located at U14 - on board. Through the USB interface, code is downloaded to the ISP. The code is then programed into the Mote by the ISP.

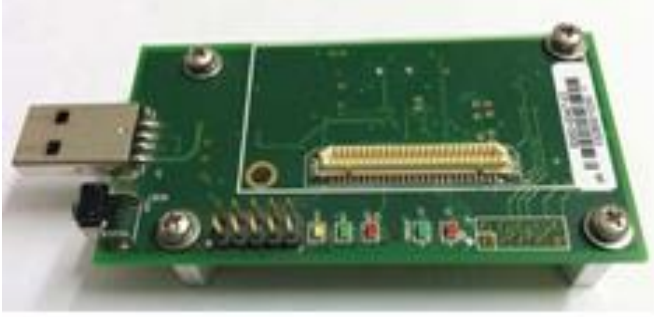

Fig 6.MIB520 Board

\section{n. Mote Programming Using the MIB520}

Mote Works TinyOS must be installed on your host PC in order to program the Motes. The MIB520 connects to the IRIS, MICAz, and MICA2 Motes for UISP programming from a USB-connected host PC. 


\section{o. MoteView}

MoteView is intended to serve as a client-side interface between a user and a network of wireless sensors. MoteView gives you the tools you need to make deployment and monitoring easier. It also makes connecting to a database, analyzing, and graphing sensor readings simple.

Fig 7. depicts a three-part framework for deploying a sensor network system. The Mote layer, often known as a sensor mesh network, is the first component. The Motes are designed to do a specific purpose using $\mathrm{XMesh} /$ TinyOS firmware ("application"), such as microclimate monitoring, asset tracking, intruder detection, and so on. Data logging and database services are provided by the second layer, or Server tier. Sensor readings arrive at this layer and are recorded on a server or Stargate by the base station (e.g., MIB510, MIB520, MIB600, or Stargate). The client tier is the third component, and it consists of software tools that show and interpret sensor data through visualization, monitoring, and analysis. The purpose of this document is to explain the features of MoteView and to provide information on the supported Mote layer applications, Mote platforms, and sensor boards.

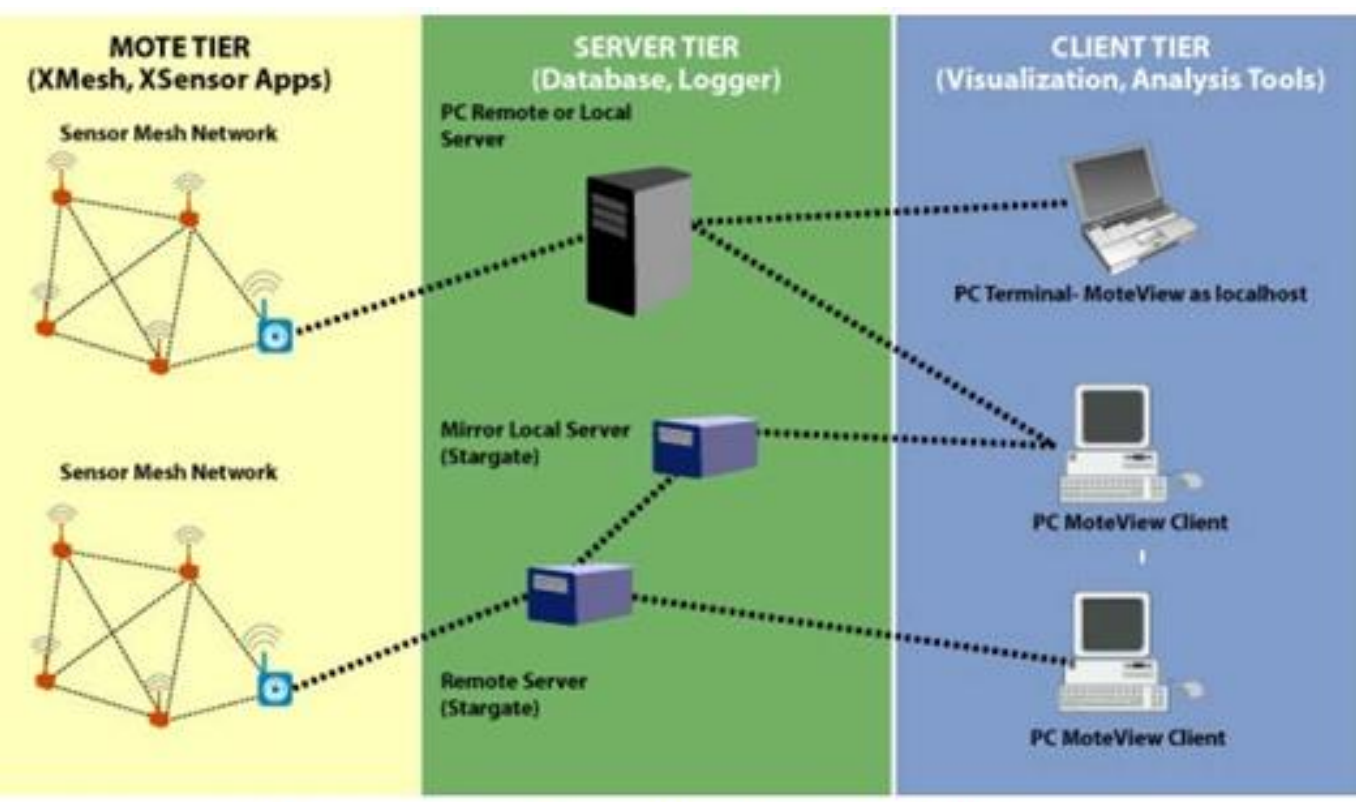

Fig 7. Software framework for a wireless sensor network

\section{p. Supported Sensor Boards and Mote Platforms}

All of Crossbow's sensor and data collection boards, as well as the MICA2, MICA2DOT, and MICAz processor/radio platforms, are supported by MoteView.

\section{q. Supported Mote Software Applications}

Crossbow's multi-hop mesh networking protocol, XMesh, has low-power listening, time synchronization, sleep modes, any-to-base, and base-to-any routing, among other features. XMesh applications are supported on all of our sensor and data collecting boards. 


\section{r. MoteConfig}

MoteConfig is a Windows-based graphical user interface (GUI) tool for programming Motes. This tool allows you to configure and download pre-compiled XMesh/TinyOS firmware apps to your Motes. The user can configure the Mote ID, Group ill, RF channel, and RF power with MoteConfig. The feature of over-the-air programming, which is available on every XMesh-based firmware, can also be enabled by the user. As part of the MoteView installation, high-power and low-power XMesh apps are offered for each sensor board and platform manufactured by Crossbow.

Depending upon the application's nature and its usage, our ease and the end user's ease we selected the following software tools to develop our application.

\section{a ASP}

ASP was used for building standards-based websites with HTML5, CSS3, and JavaScript.

\section{b Microsoft SQL Server}

MSSQL was chosen for use in web applications and as a key component of the popular LAMP open source web application software stack (and other 'AMP' stacks). "Linux, Apache, MSSQL, and Perl/PHP/Python" is an abbreviation for "Linux, Apache, MSSQL, and Perl/PHP/Python." MSSQL is frequently used in freesoftware open-source projects that require a full-featured database management system.

\subsubsection{Implementation}

Fig 8. gives a better understanding of our work

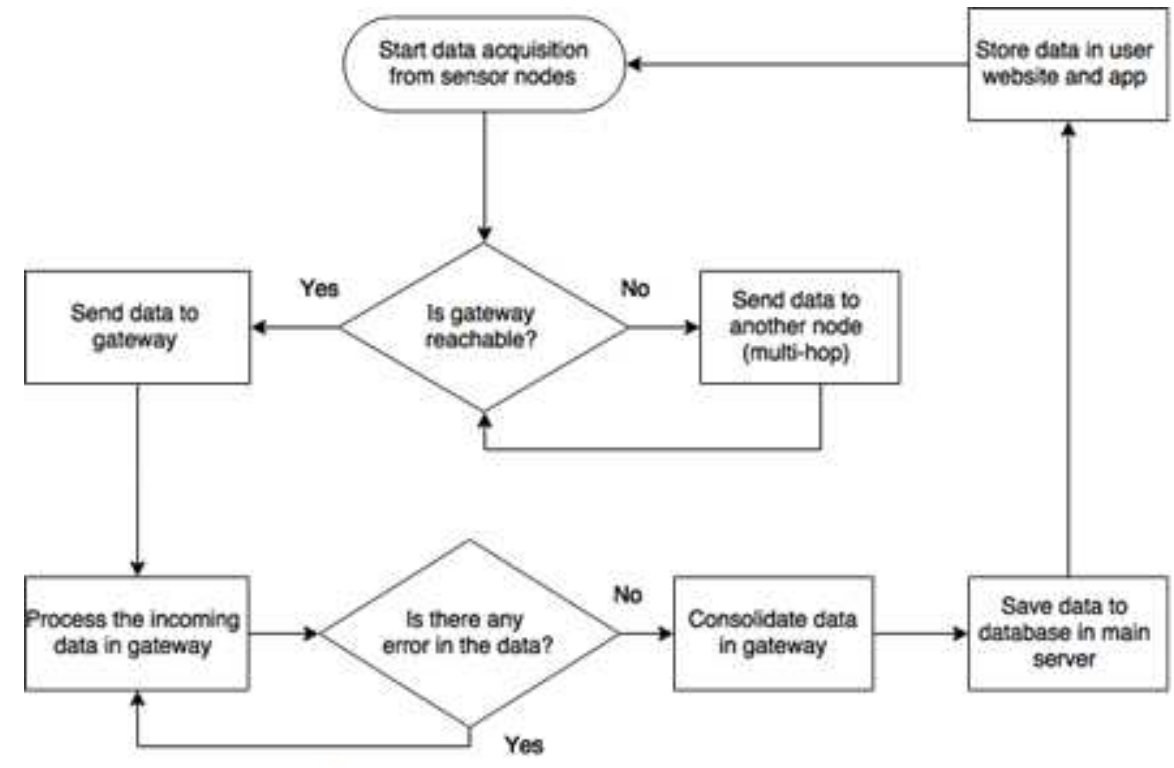

Fig 8. Data Flow diagram of EMAS 


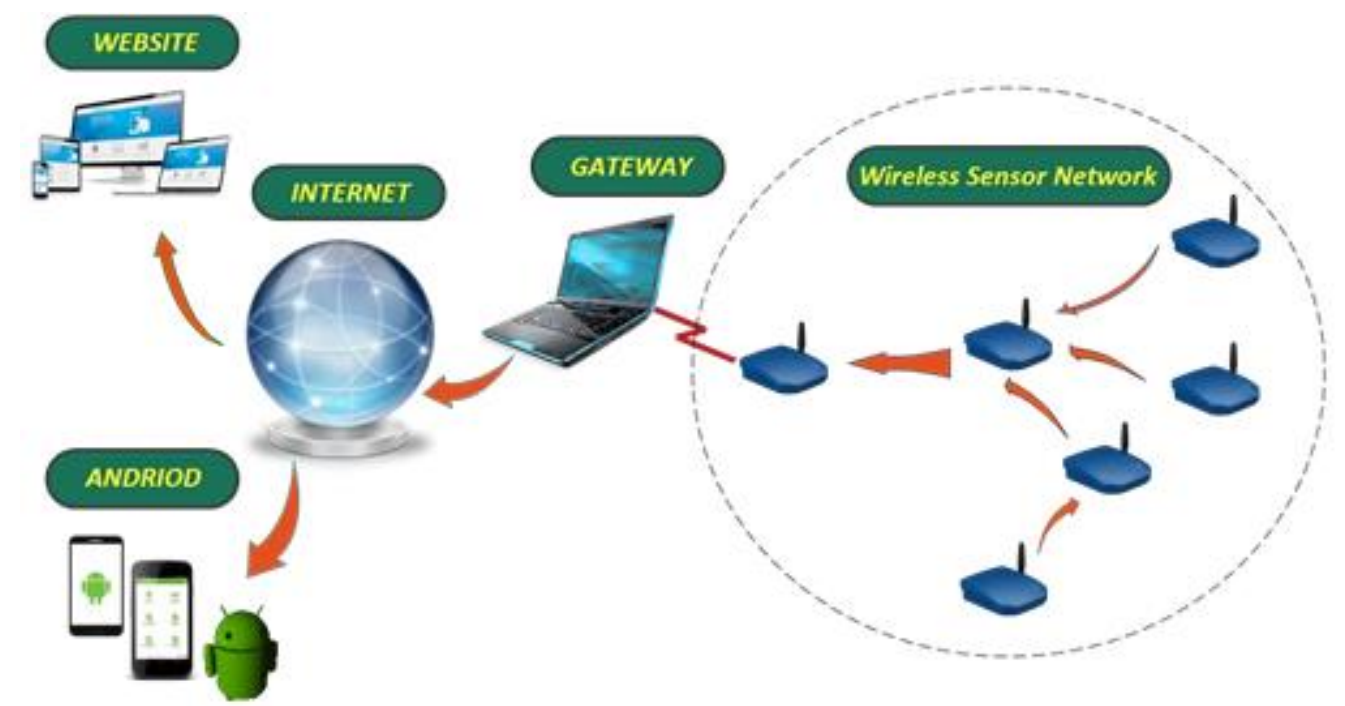

Fig 9. System Diagram

Our proposed model is designed for Sending of data from the sensor node to the gateway node, as described in fig. 9. The features include Saving of data in the database by the gateway node. It can also give the option of checking with the Database is updating the website or not and also Testing whether the project is properly linked up or not.

Integrating wireless sensors into EMAS is quite a complicated and sensitive process. To integrate the sensors into EMAS, the nodes are supposed to follow the architecture that can readily be assimilated into mainstream architectures. Wireless motes are programmed in such a way that it produces useful results. Figure 10 shows the methodology of implementation as 1) Data acquisition 2) Data collection 3) Data Retrieval. The application is designed to help the user to monitor the situation where the EMAS is installed. Application monitors the data, collect it, sends to the database the application then alerts the user about the changes happening in the environment.

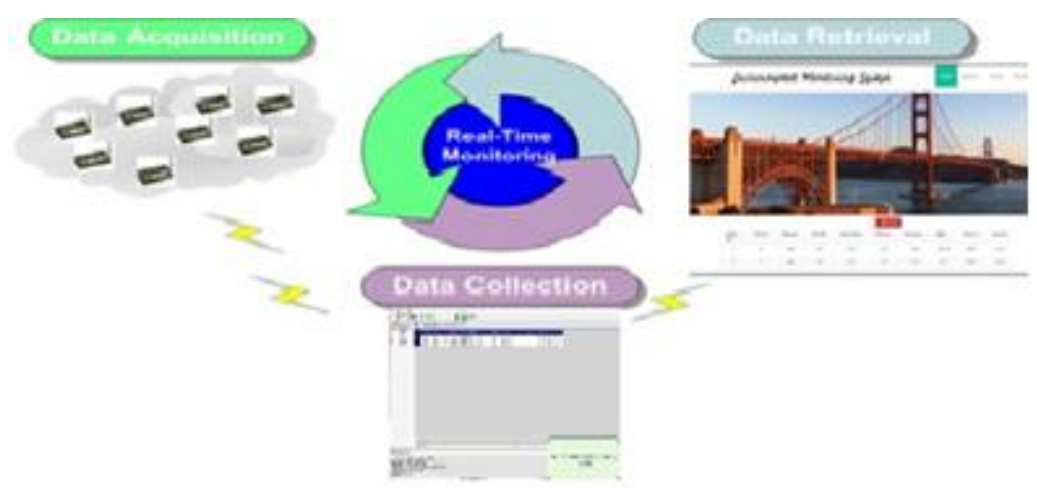

Fig 10. Data Cycle of EMAS 


\subsubsection{Data acquisition}

We have used UC Berkley / Crossbow IRIS XM2110 motes with MTS420 purchase boards mounting on it in our implementation. Atmel Atmega128 microcontroller running at 7, $3728 \mathrm{MHz}$ with a significant potion of permanent memory $(512 \mathrm{~KB})$. The system uses normal battery cells as power source. A" MIB520 USB Interface Board" is used as sensor peripheral for the sensor node. There are six nodes deployed in the system. The sensor panel has the following components: light picture resistor (Clairex CL9P4L), thermistor (YSI 44006), sensor of humidity or temperature, barometric pressure or sensor of temperature, light sensor, two-axis accelerometer, global positioning system (GPS). MDA 100 is the actuation node that has been deployed. The actuation node receives messages from the $\mathrm{CU}$ and consequently affects the operation of the EMAS. It is then necessary to pass the messages sent by the temperature sensor to one of the heating / cooling controlling EMAS Systems. All sensor motes run TinyOS, a sensor network-specific operating system. It has a programming model based on components, supplied by the language of nesC [17][18]. TinyOS produces information packets and then places them on the network using the TinySDN component programming interface [19]. It lets the setting of low-level hardware features to achieve low-power circumstances of sleep. TinyOS summarizes all hardware resources as parts [20].

\subsubsection{Data Collection}

The radio has a highly delicate receiver that can be interfered with from another IRIS node by an adjoining local oscillator. Each sensor operates in a single-hop broadcast network as a transmit-only device. The node uses the temperature sensor to sample the temperature data on the sensor board periodically, create a set of entries and schedule a transmission if required numbers are available. The data that processes the data and transmits it to EMAS is acquired at the control unit. As processing process, the control system can decide to transmits a signal to the actuation node in order to induce an actuation. In the data collection process, after converting it to engineering units from raw values, Java was used to send all the data received from nodes in real time to online database. With the dense deployment of sensor nodes, high spatial resolution for the physical phenomenon can be achieved. MS-SQL was used to create the database. MS-SQL [21] is a relational database management system developed by Microsoft. It stores the data that is collected from nodes.

\subsubsection{Data Retrieval}

Once the data is stored in the database, any time, from any device, it can be accessed from anywhere. Users can communicate in two distinct ways with the scheme. The users can access the information remotely, as it appears in the HVAC control system's database. Control system administrators can set up permissions on what remote users can change. Users on the site can have more direct control and greater access rights. The user can view various information, such as humidity and temperature, stored in the database. The node sent data is stored in the respective nodeID, showing the readings of humidity, temperature, pressure, and accelerometer. 


\section{Results and Findings}

Testing of EMAS was done from 5 sensor nodes deployed twice at different location of the subject area.

The experiment was repeated several times to get an average value-per-location giving 10 readings in total.

The data types which were collected for our proposed EMAS model are as follows:

a Temperature in ${ }^{\circ} \mathrm{C}$,

b Barometric pressure in mbar,

c Light in lux,

d Seismic vibrations in $\mathrm{x}$ and $\mathrm{y}$ coordinates in $\mathrm{mg}$,

e Humidity in percentage.

Table 7. shows the original data collected Some of these data types show high variations from one reading to another while some of them do not vary at all as shown in figure 11 .

\begin{tabular}{|l|l|l|l|l|l|}
\hline$[2020 / 07 / 18 ~ 18: 52: 48]$ MTS420 [sensor data converted to engineering] \\
\hline Humidity: & Temp: & Light: & $\begin{array}{l}\text { X-axis } \\
\text { Accel: }\end{array}$ & $\begin{array}{l}\text { Y-axis } \\
\text { Accel: }\end{array}$ & Pressure: \\
\hline$\%$ & ${ }^{\circ} \mathrm{C}$ & lux & $\mathrm{mg}$ & $\mathrm{mg}$ & mbar \\
\hline 89 & 33 & 66.01 & 600 & 380 & 991.682556 \\
\hline 86 & 33 & 66.01 & 720 & 420 & 991.589172 \\
\hline 72 & 33 & 53.13 & 680 & 400 & 991.868713 \\
\hline 71 & 32 & 62.79 & 700 & 400 & 991.77594 \\
\hline 64 & 32 & 42.09 & 740 & 400 & 991.755066 \\
\hline 54 & 32 & 69.23 & 720 & 380 & 991.557861 \\
\hline 50 & 32 & 62.79 & 740 & 380 & 991.650635 \\
\hline 43 & 32 & 62.79 & 740 & 380 & 991.743408 \\
\hline 40 & 31 & 66.01 & 780 & 400 & 991.7323 \\
\hline 37 & 32 & 56.35 & 760 & 380 & 991.86676 \\
\hline 35 & 32 & 56.35 & 760 & 380 & 991.865713 \\
\hline 34 & 31 & 56.35 & 760 & 360 & 991.337594 \\
\hline
\end{tabular}

Table 7. MTS420CA Sensor board data readings

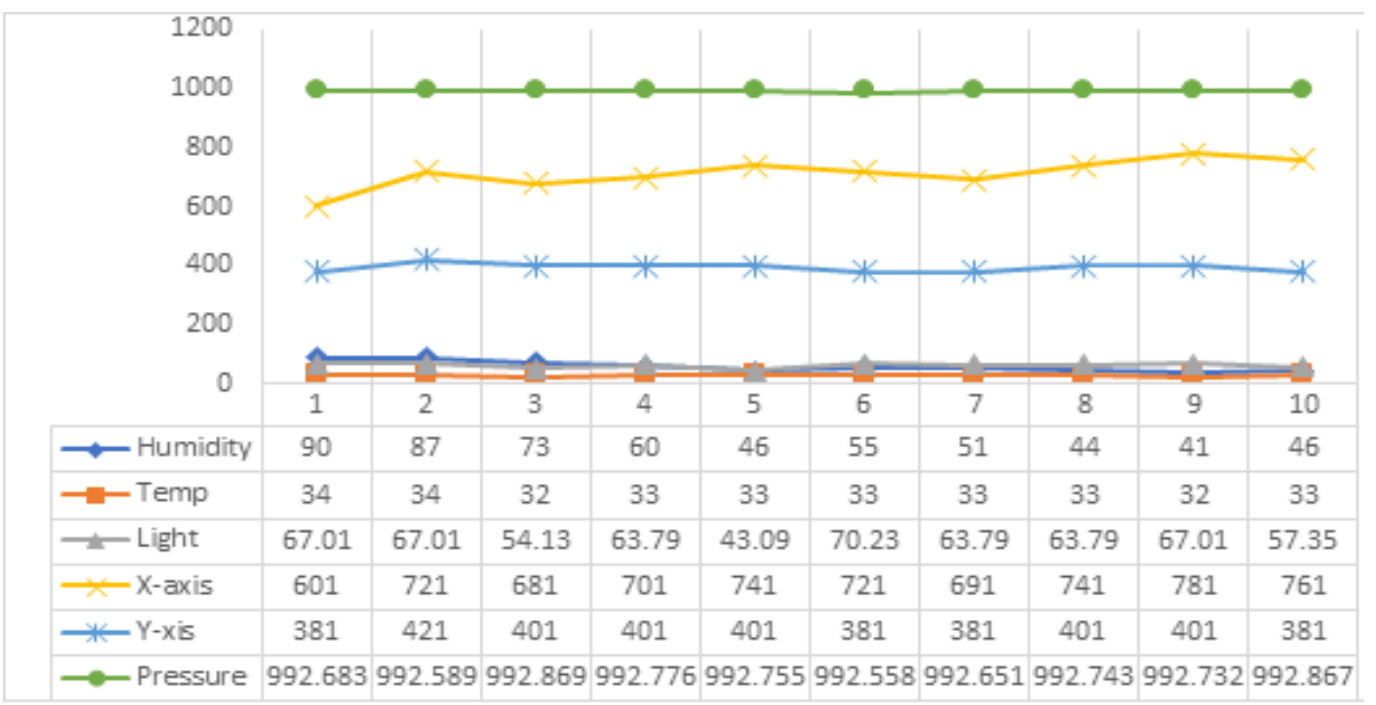

Fig 11. The Data Variation Pattern 
These variation pattern in data types can state that at what distance, locations and environments (Indoor, Outdoor) should the sensor nodes be deployed in order to get the full coverage of the area.

For example, in case of Barometric pressure the values remain almost constant (compared to the data value) while accelerometer can give substantial variations. On the other hand, in the case of temperature, humidity and light the variation is large as compared to the data value itself as shown in Fig12

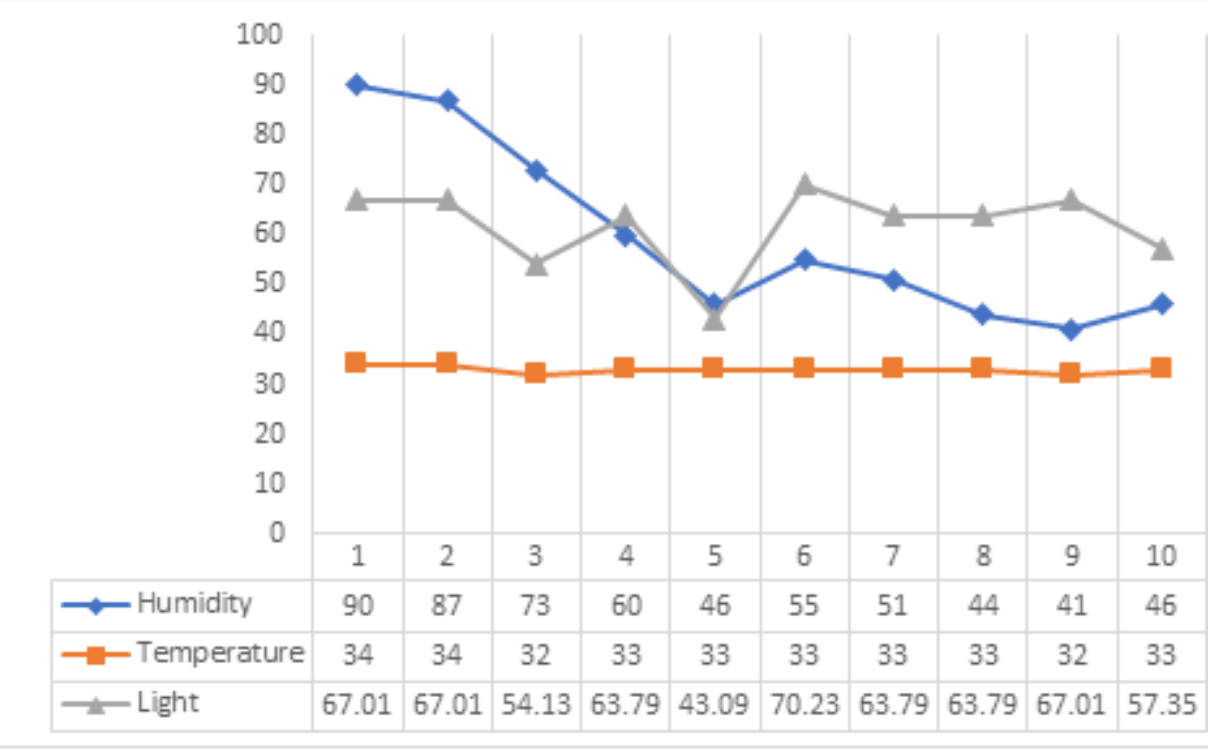

Fig 12.The data variation pattern of lower data values

This implies that in the placement of sensor nodes the coverage of the sensing should also be considered. Redundant nodes can cause unnecessary utilization of network energy. The figure 13 shows the relationship of data types extracted from table 7. In this result the standard deviation of the data set can determine the number of sensor nodes required for the correct coverage of the area. The higher the value the greater number of sensor nodes, or the higher the value the closer the sensor nodes are required to be placed.

The above results can give a good estimation of coverage area of an EMAS system. However, the distances between the nodes also affect the multihop communication within the network for transferring the data from a sensor node to the sink.

Therefore, the communication distance which in turn also depend on many factors (Multipath fading, Signal strength, electronic type etc.), should also be considered while deploying the sensor nodes.

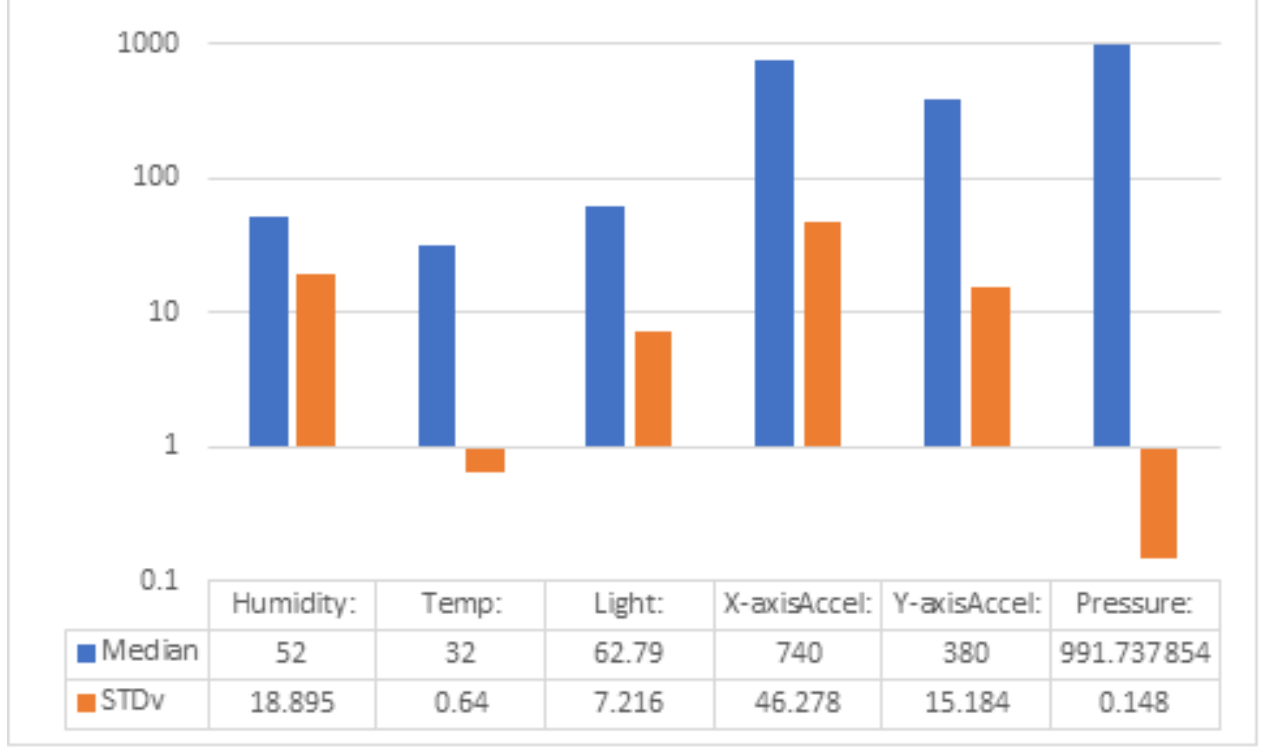

Fig13. The relationship of data types 


\subsection{Challenges}

- Power management: This is vital to long term operation, especially when monitoring remote and hostile environments is required. Harvesting schemes, cross layer protocols, and new power storage devices are identified as possible solutions to improve the lifespan of the sensors.

- Scalability: A network of wireless sensors can cater for thousands of nodes. Current environment WSN sets out to use tens to hundreds of nodes. Therefore, it is necessary to prove that the theoretical solutions available are suitable for a large real WSN.

- Remote management: Systems installed at isolated locations cannot be accessed frequently, therefore a common remote access protocol is required to run, control, reprogram and configure the WSN, regardless of the manufacturer.

- Standardization: IEEE 802.15.4 provides a millstone in attempts to standardize. Though in practice, compatibility between off the shelf modules is very low. In order to reduce costs and increase the options available, it is necessary to define standard interfaces to allow interoperability between different vendor modules.

- Mesh routing support: The topologies of mesh networks can provide both multi-hop and variety of routes. A routing protocol to support multi-hop mesh networks is therefore crucial, taking into account the network's very limited features

\section{Conclusions}

The Internet of Things is a promising progress and is currently being used in a range of applications requiring minimum human intervention. We have shown how wireless sensors and actuators can be incorporated into the current EMAS structure. An embedded architecture can be coupled with legacy systems to enable future systems to be deployed, provided energy can be scavenged for perimeter sensor nodes or battery life expanded over several years, which means that the networks will have to be energy saving rigorously. Wireless sensor networks are used in this study job and their techniques and norms were performed. Some of the most recent environmental monitoring projects were analyzed with real deployments and the conclusions were used to identify the challenges to be addressed. One thing is for sure that deployment becomes simpler than ever, and it would be as easy as plug-n-play in future moments when technology progresses to detect any physical characteristic. 


\section{Declarations}

\section{Funding}

No Funding

\section{Conflicts of interest}

We have no conflicts of interest to disclose.

\section{Availability of data and material}

All data is available with Authors

\section{Code availability}

Not Applicable

\section{References}

1. Ferdoush, Sheikh, and Xinrong Li. "Wireless sensor network system design using Raspberry Pi and Arduino for environmental monitoring applications." Procedia Computer Science 34 (2014): 103-110.

2. Othman, Mohd Fauzi, and Khairunnisa Shazali. "Wireless sensor network applications: A study in environment monitoring system." Procedia Engineering 41 (2012): 1204-1210.

3. Singh, Shio Kumar, M. P. Singh, and D. K. Singh. "A survey of energy-efficient hierarchical clusterbased routing in wireless sensor networks." International Journal of Advanced Networking and Application (IJANA) 2.02 (2010): 570-580.

4. Rault, Tifenn, Abdelmadjid Bouabdallah, and Yacine Challal. "Energy efficiency in wireless sensor networks: A top-down survey." Computer Networks 67 (2014): 104-122.

5. Arampatzis, Th, John Lygeros, and Stamatis Manesis. "A survey of applications of wireless sensors and wireless sensor networks." Proceedings of the 2005 IEEE International Symposium on, Mediterrean Conference on Control and Automation Intelligent Control, 2005.. IEEE, 2005.

6. Akkaya, Kemal, and Mohamed Younis. "A survey on routing protocols for wireless sensornetworks." Ad hoc networks 3.3 (2005): 325-349.

7. Abbasi, Ameer Ahmed, and Mohamed Younis. "A survey on clustering algorithms forwireless sensor networks." Computer communications 30.14-15 (2007): 2826-2841.

8. Jindal, V. "History and architecture of Wireless sensor networks for ubiquitous computing." International Journal of Advanced Research in Computer Engineering Technology (IJARCET) 7.2 (2018): 214-217.

9. Ruiz-Garcia, Luis, et al. "A review of wireless sensor technologies and applications inagriculture and food industry: state of the art and current trends." sensors 9.6 (2009): 4728-4750. ACM, 2013

10. Patil, K. A., and N. R. Kale. "A model for smart agriculture using IoT." 2016 International Conference on Global Trends in Signal Processing, Information Computing and Communication (ICGTSPICC). IEEE, 2016.

11. Hart, Jane K., and Kirk Martinez. "Environmental sensor networks: A revolution in the earth system science?." Earth-Science Reviews 78.3-4 (2006): 177-191

12. Healy, William M. "Lessons learned in wireless monitoring." Ashrae Journal 47.10 (2005): 54.

13. Jang, Won-Suk, William M. Healy, and Mirosl aw J. Skibniewski. "Wireless sensor networks as part of a web-based building environmental monitoring system." Automation in Construction 17.6 (2008): 729736.

14. Agarwal, Yuvraj, et al. "Duty-cycling buildings aggressively: The next frontier in HVACcontrol." Proceedings of the 10th ACM/IEEE International Conference on Information Processing in Sensor Networks. IEEE, 2011.

15. Stoleru, Radu, John A. Stankovic, and Sang H. Son. "Robust node localization for wireless sensor networks." Proceedings of the 4th workshop on Embedded networked sensors. ACM, 2007.

16. Banerjee, Suman, and Samir Khuller. "A clustering scheme for hierarchical control inmulti-hop wireless networks." IEEE INFOCOM. Vol. 2. INSTITUTE OF ELECTRICAL ENGINEERS INC (IEEE), 2001. 
17. Levis, Philip, et al. "TOSSIM: Accurate and scalable simulation of entire TinyOS applications." Proceedings of the 1st international conference on Embedded networked sensor systems. ACM, 2003

18. Hempstead, Mark, Matt Welsh, and David Brooks. "TinyBench: The case for a standardized benchmark suite for TinyOS based wireless sensor network devices." 29th Annual IEEE International Conference on Local Computer Networks. IEEE, 2004.

19. de Oliveira, Bruno T., and Cintia B. Margi. "TinySDN: Enabling tinyOS to softwaredefined wireless sensor networks.” XXXIV Simp'osio Brasileiro de Redes de Computadores (2016): 1229-1237

20. Levis, Philip, et al. "TinyOS: An operating system for sensor networks." Ambient intelligence. Springer, Berlin, Heidelberg, 2005. 115-148.

21. "MySQL AB: The world's most popular open source database" www.mysql.com

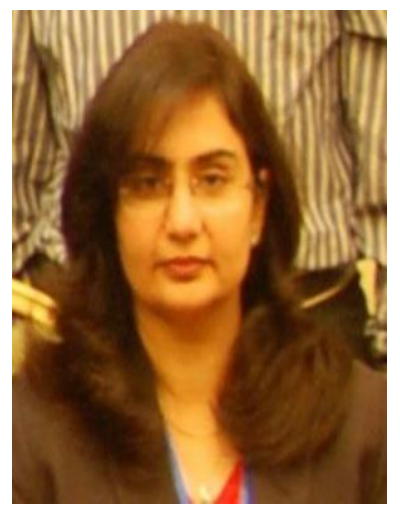

Rabia Noor Enam is serving as associate professor in the department of computer engineering at Sir Syed University of Engineering \& Technology, Karachi, Pakistan. Dr. Rabia received the Bachelors and Masters degree in Applied Mathematics from Karachi University in 1991 and 1992 respectively. She did her Bachelors in Computer Engineering from N.E.D. University in 1997 and completed her MS in Computer Networks Engineering in 2002 from SSUET. She completed her PhD in Computer Engineering from SSUET. The title of her PhD thesis was "Energy Efficient Adaptive Data Aggregation in Cluster Based Wireless Sensor Networks" Networks". Her research interests are in communication protocols and aggregation techniques in Wireless Sensor Networks. Up to date, there are 19 articles/papers in the list of her publications, which includes several ISI indexed impact factor journals and International conferences.

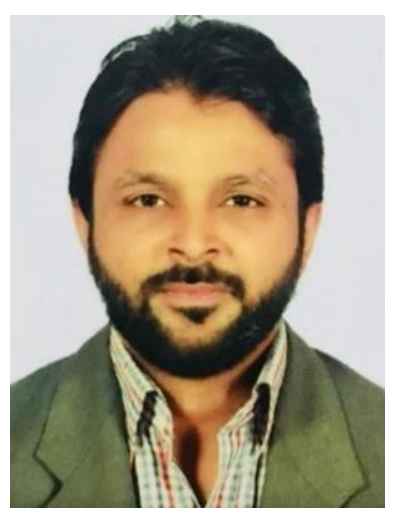

Muhammad Tahir received the BS degree in computer engineering from Sir Syed University, M.E. degree in Computer System from NED University and PhD in Information Science from University of Roma Tor Vergata. He is currently Associate Professor in Sir Syed University of Engineering and Technology, Karachi. His research interests include IP Switches/Routing, IPv4 Protocol, Firewall, IoT, Security Cryptography and Wireless Networks. 

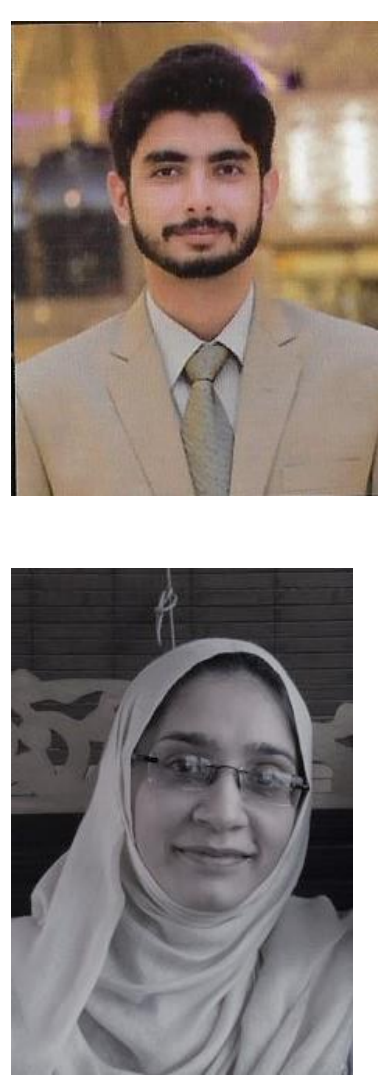

Syed Muhammad Nabeel Mustafa received his degree in software engineering from Sir Syed University of Engineering and Technology, Karachi in 2019. He is pursuing a post graduate degree in Data Science for NED University, Karachi. The author has research experience in Artificial intelligence, Data Science and Internet of things (IoT). He has a number of publications to his name.

Rukaiya Rukaiya received the B.S. degree in computer engineering from the Sir Syed University of Engineering and Technology (SSUET), Karachi, Pakistan, in 2011, and the M.S. degree in computer engineering with a specialization in computer networks from SSUET, in 2015. She is currently pursuing the Ph.D. degree in computer engineering with the National University of Sciences and Technology (NUST), Islamabad, Pakistan. She worked as a Research Associate with the Office of Research, Innovation and Commercialization (ORIC), SSUET. She is also serving as a Lecturer for the Department of Computer Engineering, SSUET. Her research interests include MAC and routing protocols, wireless adhoc networks, mission critical tactical networks, and network security. 